ESAIM: COCV 19 (2013) 301-316

DOI: $10.1051 / \mathrm{cocv} / 2012009$
ESAIM: Control, Optimisation and Calculus of Variations

www.esaim-cocv.org

\title{
EXACT CONTROLLABILITY OF THE 1-D WAVE EQUATION FROM A MOVING INTERIOR POINT
}

\author{
Carlos Castro ${ }^{1}$
}

\begin{abstract}
We consider the linear wave equation with Dirichlet boundary conditions in a bounded interval, and with a control acting on a moving point. We give sufficient conditions on the trajectory of the control in order to have the exact controllability property.
\end{abstract}

Mathematics Subject Classification. 93B05, 93B07, 35L05.

Received 15 March, 2012. Revised 9 September, 2011.

Published online 3 July 2012.

\section{INTRODUCTION}

We consider the one-dimensional linear wave equation, on a finite interval domain $(0, L)$, with an interior control $f$ which acts on a single moving point $x=\gamma(t)$,

$$
\begin{cases}u_{t t}-u_{x x}=f(t) \delta_{\gamma(t)}(x), & \text { in } 0<x<L, 0<t<T, \\ u(0, t)=u(L, t)=0, & \text { in } \quad 0<t<T, \\ u(x, 0)=u^{0}(x), u_{t}(x, 0)=u^{1}(x) & \text { in } 0<x<L .\end{cases}
$$

Here, $\delta_{\gamma(t)}$ represents the Dirac measure on $x=\gamma(t)$ and the function $\gamma$ describes the trajectory in time of the location of the control. We assume that the function $\gamma:[0, T] \rightarrow(0, L)$ is piecewise $C^{1}$ in $[0, T]$.

We are interested in the following exact controllability problem: Given $T>0$, some initial data $\left(u^{0}, u^{1}\right)$ and final data $\left(v^{0}, v^{1}\right)$, find a control $f$ such that the solution $u$ of (1.1) satisfies

$$
u(x, T)=v^{0}(x), \quad u_{t}(x, T)=v^{1}(x), \quad \forall x \in(0, L) .
$$

Let us briefly describe some related results and the main motivation of this problem.

When the control acts in an interior open set $\omega$ or one of the extremes of the domain $(0, L)$, it is well known that the corresponding exact controllability property of the wave equation holds, for some sufficiently large time $T$ (see [12]). On the other hand, in most practical situations, the support of the control is required to be very small compared to the total size of the domain $(0, L)$ and therefore it is very natural to consider a limit situation where the subinterval $\omega$ is reduced to a single point $\gamma \in(0, L)$. It turns out that the controllability property of system (1.1) depends on the location of $\gamma$. Indeed, it can be shown that this property holds if and only if the

Keywords and phrases. Exact controllability, wave equation, pointwise control.

1 Dep. Matemática e Informática, ETSI Caminos, Canales y Puertos, Universidad Politécnica de Madrid, 28040 Madrid, Spain. carlos.castro@upm.es 
only eigenfunction of the Laplacian with homogeneous Dirichlet boundary conditions and vanishing on $x=\gamma$ is the identically zero one (see $[1,11,13]$ or the more recent Ref. [5], for example). In the sequel, the points $\gamma$ for which this spectral property is satisfied will be referred to as strategic points.

The property of $\gamma$ being strategic is difficult to establish in practice since it is extremely unstable. In fact $\gamma \in(0, L)$ is strategic if and only if it is irrational with respect to the length of the interval $L$. Consequently, controllability properties over points are hard to use in practice.

To overcome this difficulty one may consider controls supported on moving points $\{\gamma(t)\}_{0 \leq t \leq T}$, as suggested in [13] (the same strategy is analyzed in the context of feedback stabilization in [2]). The main advantage of moving controls is that it is easy to construct trajectories $\{\gamma(t)\}_{0 \leq t \leq T}$ for which the strategic property holds for $\gamma(t) \in(0, L)$ a.e. in $t \in[0, T]$. For example, this is the case when we assume that the control is located at a point that moves in time with constant velocity. In this case, $\gamma(t)$ is irrational, and therefore strategic, a.e. in $t \in[0, T]$. Therefore, the exact controllability is likely to hold for such moving controls. The aim of this work is to show that this is indeed the case under suitable conditions on the function $\gamma(t)$.

This problem has been previously studied in [8] and [9] where some sufficient conditions on the curve are given. Here we introduce a new approach that provides the exact controllability property for a larger class of curves. In contrast, our approach provides controls in $\left(H^{1}(0, T)\right)^{\prime}$, the dual space of $H^{1}(0, T)$. We do not know if it can be adapted to obtain $L^{2}$-controls, at least in an easy way, due to the fact that the system is not autonomous.

It is worth noting that in the context of parabolic equations a similar situation appears. We refer to $[1,4,8,13]$ and the references therein for a detailed analysis of this related problem.

The rest of this paper is divided as follows: in Section 2 we state the main results, namely the existence of solutions for system (1.1) in suitable functional spaces and the exact controllability property. Both results can be reduced, by classical duality arguments, to some suitable regularity and observability estimates for the uncontrolled wave equation respectively. In Section 3 we give some examples of curves for which the exact controllability property holds. In Section 4 we give the proof of the observability estimates. Finally, in Section 6 we study the existence of weak solutions of (1.1) and in Section 7 we briefly comment the HUM method that allows to obtain the controllability result from the observability inequality.

\section{MAIN RESULTS}

We first state the well-posedness of system (1.1). The control $f(t)$ in $(1.1)$ is assumed to belong to $\left(H^{1}(0, T)\right)^{\prime}$, the dual space of $H^{1}(0, T)$, and the initial data $\left(u^{0}, u^{1}\right)$ in the class

$$
\left(u^{0}, u^{1}\right) \in L^{2}(0, L) \times H^{-1}(0, L)
$$

We define the weak solutions of system (1.1) by transposition (see [10]). To do that let $\psi \in L^{1}\left(0, T ; L^{2}(0, L)\right.$ ) be a function and consider the non-homogeneous adjoint wave equation

$$
\begin{cases}\varphi_{t t}-\varphi_{x x}=\psi(x, t) & \text { in } 0<x<L, 0<t<T, \\ \varphi(0, t)=\varphi(L, t)=0 & \text { in } 0<t<T, \\ \varphi(x, T)=\varphi_{t}(x, T)=0, & \text { in } 0<x<L, 0<t<T .\end{cases}
$$

It is well known that system (2.3) admits a unique solution $\varphi$ of (2.3) in the class

$$
\varphi \in C\left([0, T] ; \quad H_{0}^{1}(0, L)\right) \cap C^{1}\left([0, T] ; \quad L^{2}(0, L)\right) .
$$


Multiplying the equations in (1.1) by $\varphi$ and integrating by parts we easily obtain, at least formally, the following identity:

$$
\begin{aligned}
& \left\langle u^{1}(x), \varphi(x, 0)\right\rangle_{1}-\int_{0}^{L} u^{0}(x) \varphi_{t}(x, 0) \mathrm{d} x+\langle f, \varphi(\gamma(t), t)\rangle_{1}^{t} \\
& =\int_{0}^{T} \int_{0}^{L} \psi(x, t) u(x, t) \mathrm{d} x \mathrm{~d} t, \text { for all } \psi \in L^{1}\left(0, T ; L^{2}(0, L)\right),
\end{aligned}
$$

where $\langle\cdot, \cdot\rangle_{1}$ and $\langle\cdot, \cdot\rangle_{1}^{t}$ denote the duality products in $H_{0}^{1}(0, L)$ and $H^{1}(0, T)$ respectively.

We adopt identity (2.5) as the definition of solutions of (1.1), in the sense of transposition.

The following result establishes the existence of solutions for system (1.1).

Theorem 2.1. Assume that $\gamma:[0, T] \rightarrow(0, L)$ is in the class $\gamma \in C^{1}([0, T])$ piecewise, i.e. there exists a partition of $[0, T], 0=t_{0}<t_{1}<\ldots<t_{n}=T$, such that $\gamma \in C^{1}\left(\left[t_{i}, t_{i+1}\right]\right)$ for all $i=0, \ldots, n-1$. Assume also that this partition can be chosen in such a way that $1-\left|\gamma^{\prime}(t)\right|$ does not change the sign in $t \in\left[t_{i}, t_{i+1}\right]$, for all $i=0,1, \ldots, n-1$. Given any initial data $\left(u^{0}, u^{1}\right) \in L^{2}(0, L) \times H^{-1}(0,1)$ and $f \in\left(H^{1}(0, T)\right)^{\prime}$, there exists an unique solution $u$ of (1.1), in the sense of transposition, in the class

$$
u \in C\left([0, T] ; L^{2}(0, L)\right), \quad u_{t} \in L^{2}\left(0, T ; H^{-1}(0, L)\right) .
$$

Moreover, there exists a one-to-one correspondence between the data and the solution in the given spaces.

Concerning the exact controllability problem of system (1.1) we have to consider further hypothesis on the curve $\gamma$.

Definition 2.2. We say that a curve $\gamma:[0, T] \rightarrow(0, L)$ is an 'admissible trajectory' if system (1.1) is exactly controllable, i.e. for any initial data $\left(u^{0}, u^{1}\right) \in L^{2}(0, L) \times H^{-1}(0, L)$ and final data $\left(v^{0}, v^{1}\right) \in L^{2}(0, L) \times H^{-1}(0, L)$, there exists a control $f \in\left(H^{1}(0, T)\right)^{\prime}$ such that the solution $u$ of (1.1) satisfies (1.2).

Remark 2.3. When dealing with control problems for the wave equation, the finite speed of propagation affects to the minimal time interval necessary for controllability $\left[0, T_{\min }\right]$. The value $T_{\min }$ is usually given by an optical geometric condition requiring that any ray, starting anywhere in the domain and with any initial direction, must meet the controllability zone before the time $T_{\min }$. According to this, an admissible trajectory requires both geometric constraints and a condition on the length of the time interval $[0, T]$, that must be sufficiently large.

Theorem 2.4. Assume that $\gamma \in C^{1}([0, T])$ piecewise and it satisfies the following hypothesis:

1. There exists constants $c_{1}, c_{2}>0$ and a finite number of open subintervals $I_{j} \subset[0, T]$ with $j=0, \ldots, J$ such that, for each subinterval $I_{j}, \gamma \in C^{1}\left(I_{j}\right)$ and it satisfies the following two conditions

- $c_{1} \leq\left|\gamma^{\prime}(t)\right| \leq c_{2}$ for all $t \in I_{j}$,

- $1-\left|\gamma^{\prime}(t)\right|$ does not change the sign in $t \in I_{j}$.

We assume, without loss of generality, that there exists $j_{1}$ with $-1 \leq j_{1} \leq J$ such that $\gamma(t)$ is decreasing in $I_{j}$ for $0 \leq j \leq j_{1}$, and $\gamma(t)$ is increasing in $I_{j}$ for $j_{1}<j \leq J$. Here, $j_{1}=-1$ corresponds to the case where $\gamma(t)$ is increasing in all the subintervals $I_{j}$. Analogously, $j_{1}=J$ corresponds to the case where $\gamma(t)$ is decreasing in all the subintervals $I_{j}$.

2. For each $j=0, \ldots, J$, let $U_{j}$ be the subintervals defined as follows:

$$
U_{j}= \begin{cases}\left\{s-\gamma(s) \text { with } s \in I_{j}\right\} & \text { if } j \leq j_{1}, \\ \left\{s+\gamma(s) \text { with } s \in I_{j}\right\} & \text { if } j>j_{1} .\end{cases}
$$

Then, there exists an interval $W_{1}$ with length $\left(W_{1}\right)>2 L$ such that

$$
W_{1} \subset \overline{\bigcup_{j=0}^{J} U_{j}} \text {. }
$$


3. For each $j=0, \ldots, J$, let $V_{j}$ be the subintervals defined as follows:

$$
V_{j}= \begin{cases}\left\{s+\gamma(s) \text { with } s \in I_{j}\right\} & \text { if } j \leq j_{1}, \\ \left\{s-\gamma(s) \text { with } s \in I_{j}\right\} & \text { if } j>j_{1} .\end{cases}
$$

Then, there exists a subinterval $W_{2}$, with length $\left(W_{2}\right) \leq$ length $\left(W_{1}\right)$, such that

$$
\bigcup_{j=0}^{J} V_{j} \subset \overline{W_{2}},
$$

and

$$
V_{j} \cap V_{k}=\emptyset \text {, if } j \neq k .
$$

Then $\gamma$ is admissible in the sense of Definition 2.2.

The proof of the existence result above (Thm. 2.1) can be obtained from a suitable regularity property stated below (estimate (4.11)) by a duality argument. We refer to [10] for a general description, and [6] or [3] where this is done for similar problems. The novelty in the present situation is that the system (1.1) is not autonomous. We give the proof of Theorem 2.1 in Section 6 below.

The exact controllability property (Thm. 2.4) is also a straightforward consequence of the observability inequality (4.12) below and the Hilbert Uniqueness Method introduced by Lions in [12]. We give the proof in Section 7 . We also refer to $[3,6]$ where this method is applied for similar problems.

\section{Admissible trajectories}

In this section we show some examples of curves $\gamma$ that satisfy the hypothesis of Theorem 2.4 and therefore are admissible trajectories for the exact controllability of system (1.1), in the sense of Definition 2.2.

Example 3.1 (slowly decreasing trajectories, as plotted on the right hand side of Fig. 1). Assume that $\gamma \in$ $C^{1}([0, T])$ satisfies the following:

1. $-1 \leq \gamma^{\prime}(t) \leq-c_{1}$ for some constant $c_{1}>0$ and for all $t \in[0, T]$,

2. the time $T$ is large enough to have $T-\gamma(T)+\gamma(0) 2 L$.

Then $\gamma$ is an admissible trajectory in the sense of Definition 2.2. In fact we easily check that the hypothesis of Theorem 2.4 are satisfied with the interval $I_{0}=(0, T)$. In this case, $U_{0}=(-\gamma(0),-\gamma(T)+T)=W_{1}$ and $V_{0} \subset[\gamma(0), T+\gamma(T)]=W_{2}$. The condition $-1 \leq \gamma^{\prime}(t)$ guarantees that $V_{0}$ is a proper interval, i.e. $T+\gamma(T)-\gamma(0) \geq 0$. In fact, this inequality is easily obtained by integrating $-1 \leq \gamma^{\prime}(t)$ in $t \in(0, T)$.

Note also that, by the hypothesis 2 above, the length of $U_{0}$ is greater than $2 L$ and the second hypothesis in Theorem 2.4 holds. On the other hand, the hypothesis 3 in Theorem 2.4 is easily checked since the length of $U_{0}$ is also greater than the length of $V_{0}$ due to the fact that $\gamma$ is decreasing in $[0, T]$.

The second condition above, i.e. $T-\gamma(T)+\gamma(0)>2 L$, provides an estimate of the minimal time for controllability which turns out to be optimal in some particular cases. For example, if we take $L \geq 1, \gamma(t)=$ $L / 2-t / 4$, and $T>8 L / 5$ we have an admissible trajectory since it satisfies the conditions above. The time $T=8 L / 5$ is minimal in the sense that it is the time required for a ray starting at $x_{0}=L / 2$ with initial velocity $x^{\prime}(0)=1 / 2$, to meet the controllability zone, following the optical geometric laws (see Rem. 2.3).

Example 3.2 (slowly increasing trajectories, as plotted on the left hand side of Fig. 1). Assume that $\gamma \in$ $C^{1}([0, T])$ satisfies the following:

1. $0<c_{1} \leq \gamma^{\prime}(t) \leq 1$ for all $t \in[0, T]$,

2. the time $T$ is large enough to have $T+\gamma(T)-\gamma(0) 2 L$. 


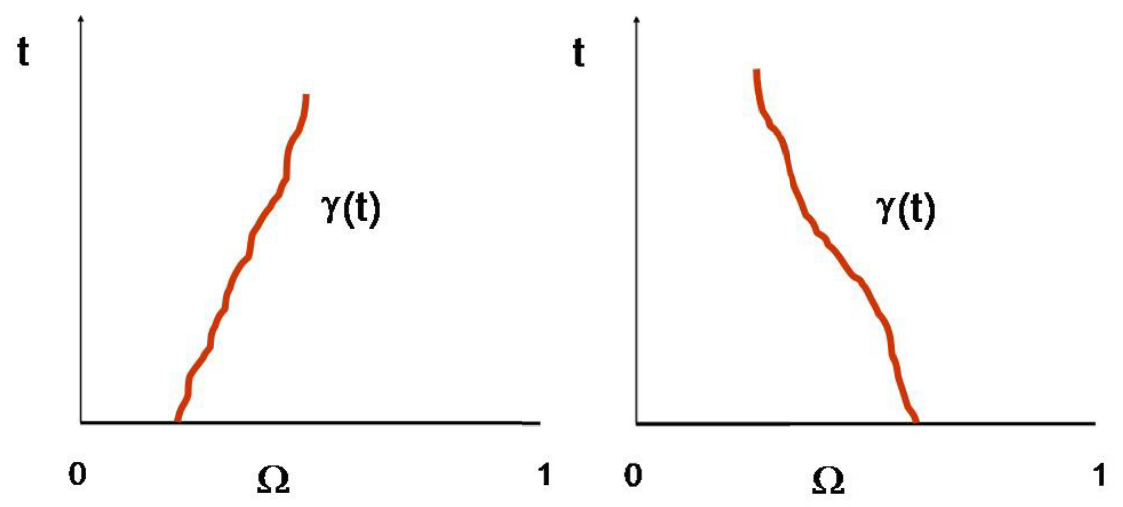

FiguRE 1. Slowly increasing (left) and decreasing trajectories that are admissible

Then $\gamma$ is admissible. Again, we easily check that the hypothesis of theorem are satisfied with the interval $I_{0}=(0, T)$.

Example 3.3 (rapidly decreasing trajectories). In this section we assume that $\gamma^{\prime}(t) \leq-1$. Obviously, such a trajectory will meet the boundary of the domain in short time and it is not expected to have an admissible trajectory $\gamma \in C^{1}\left([0, T]\right.$ ) (see Rem. 2.3). Thus, we will assume that the trajectory is piecewise $C^{1}$ and it is decreasing only in some intervals, where it is also $C^{1}$. More precisely, we assume that:

(a) there exists a family of open subintervals $I_{j}=\left(a_{j}, b_{j}\right) \subset(0, T)$ with $j=0, \ldots, J$, and the interval $\omega=$ $\left(\omega_{1}, \omega_{2}\right) \subset(0, L)$ such that $\gamma:\left(a_{j}, b_{j}\right) \rightarrow\left(\omega_{1}, \omega_{2}\right)$

(b) there exists $c_{2}>0$ such that $-c_{2}<\gamma^{\prime}(t) \leq-1$ for all $t \in\left(a_{j}, b_{j}\right)$ and for all $j=0,1, \ldots, J$;

(c) $0=a_{0}<a_{1}<\ldots<a_{J}<T$ and $0<b_{0}<b_{1}<\ldots<b_{J}=T$;

(d) $a_{j+1}-\gamma\left(a_{j+1}\right) \leq b_{j}-\gamma\left(b_{j}\right)$, for all $j=0,1, \ldots, J-1$;

(e) $b_{j+1}+\gamma\left(b_{j+1}\right) \geq a_{j}+\gamma\left(a_{j}\right)$, for all $j=0,1, \ldots, J-1$;

(f) $\gamma(0)=\omega_{2}, \gamma(T)=\omega_{1}$;

(g) $T-\gamma(T)+\gamma(0)>2 L$.

Then, $\gamma$ is admissible. In Figure 2 we show an example of such trajectory. It is interesting to note that the intervals $I_{j}$ do not cover neccesarily the whole interval $(0, T)$. This is only the case if $a_{j}=b_{j-1}$ for all $j=1, \ldots, J$. Obviously, this trajectory can be completed with piecisewise $C^{1}$ curves outside $I_{j}$ and the result still holds (see the right hand side of Fig. 2).

Let us prove that $\gamma$ satisfies the conditions of Theorem 2.4. The first condition is a direct consequence of the hypothesis (b) above. In order to check the second condition in Theorem 2.4 we define $U_{j}=\{s-\gamma(s)$ with $\left.s \in I_{j}\right\}$ and $W_{1}=\left(-\omega_{2}, T-\omega_{1}\right)$. Then by hypothesis (d) and (f),

$$
W_{1}=(-\gamma(0), T-\gamma(T)) \subset \overline{\bigcup_{j=0}^{J}\left(a_{j}-\gamma\left(a_{j}\right), b_{j}-\gamma\left(b_{j}\right)\right)}=\overline{\bigcup_{j=0}^{J} U_{j}},
$$

and by hypothesis $(\mathrm{g})$ the length of $W_{1}$ is equal or greater than $2 L$. We refer to Figure 3 for a geometrical interpretation of this condition on the intervals $U_{j}$. 

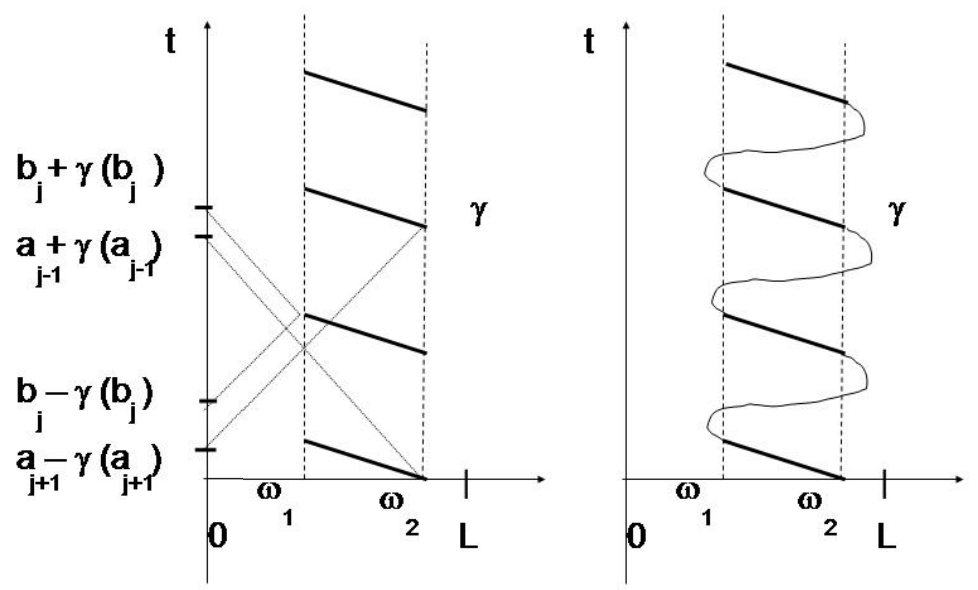

FIgURE 2. Rapidly decreasing trajectory that satisfies the conditions to be admissible. In particular we see that $a_{j+1}-\gamma\left(a_{j+1}\right) \leq b_{j}-\gamma\left(b_{j}\right)$ and $b_{j+1}+\gamma\left(b_{j+1}\right) \geq a_{j}+\gamma\left(a_{j}\right)$ (left). The result still holds if we complete the trajectory by piecewise $C^{1}$ curves in order to have a continuous trajectory (right).
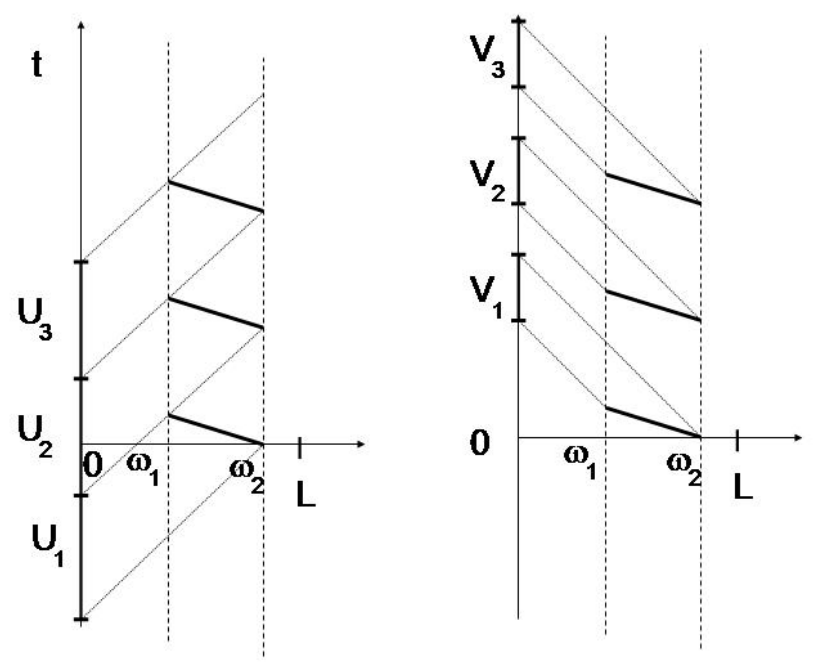

FiguRE 3. Rapidly decreasing trajectory that satisfies the conditions to be admissible. Here we show the geometrical interpretation of $U_{j}$ and $V_{j}$.

Now we check the third condition in Theorem 2.4. Define $V_{j}=\left\{s+\gamma(s)\right.$ with $\left.s \in I_{j}\right\}$ and $W_{2}=\left(b_{0}+\omega_{1}, a_{J}+\right.$ $\left.\omega_{2}\right)$. By hypothesis (c) and (e), $V_{j} \cap V_{k}=\emptyset$ if $j \neq k$ and

$$
\overline{\bigcup_{j=0}^{J}\left(b_{j}+\gamma\left(b_{j}\right), a_{j}+\gamma\left(a_{j}\right)\right)} \subset\left[b_{0}+\gamma\left(b_{0}\right), a_{J}+\gamma\left(a_{J}\right)\right] \subset \overline{W_{2}} .
$$




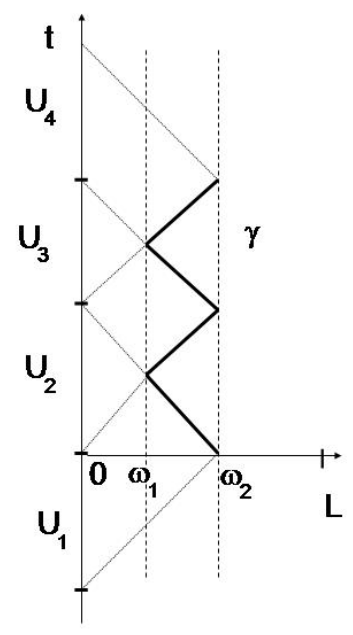

FiguRE 4. Saw-teeth trajectory that satisfies the conditions to be admissible with minimal time. Here $\omega_{1}=L^{*} / 4, \omega_{2}=L^{*} / 2$, and the time $T=L^{*}>L$.

The last inclusion is obtained from the fact that both $\gamma\left(b_{0}\right), \gamma\left(a_{J}\right) \in\left(\omega_{1}, \omega_{2}\right)$ which is deduced by hypothesis (a). On the other hand,

$$
\overline{W_{2}} \subset\left(\omega_{1}, T+\omega_{2}\right),
$$

which has the same length as $W_{1}$. Thus, the third condition in Theorem 2.4 is a direct consequence of (3.8) and (3.9).

Example 3.4 (rapidly increasing trajectories). We assume that:

(a) there exists a family of open subintervals $I_{j}=\left(a_{j}, b_{j}\right) \subset(0, T)$ with $j=0, \ldots, J$, and the interval $\omega=$ $\left(\omega_{1}, \omega_{2}\right) \subset(0, L)$ such that $\gamma:\left(a_{j}, b_{j}\right) \rightarrow \omega$;

(b) there exists $c_{2}>0$ such that $1 \leq \gamma^{\prime}(t)<c_{2}$ for all $t \in\left(a_{j}, b_{j}\right)$ and for all $j=0,1, \ldots, J$;

(c) $0=a_{0}<a_{1}<\ldots<a_{J}<T$ and $0<b_{0}<b_{1}<\ldots<b_{J}=T$;

(d) $a_{j+1}+\gamma\left(a_{j+1}\right) \leq b_{j}+\gamma\left(b_{j}\right)$;

(e) $b_{j+1}-\gamma\left(b_{j+1}\right) \geq a_{j}-\gamma\left(a_{j}\right)$ for all $j=0,1, \ldots, J-1$;

(f) $\gamma(0)=\omega_{1}, \gamma(T)=\omega_{2}$;

(g) $T+\gamma(T)-\gamma(0)>2 L$.

Then, $\gamma$ is admissible. The proof is analogous to the previous case.

Example 3.5 (saw-teeth trajectories). As we said in Remark 2.3 above, when dealing with control problems for the wave equation, the minimal time for controllability $T_{\min }$ is usually given by the a optical geometric condition. We show that for some trajectories, like in Figure 4, controllability holds for any time $T$ strictly larger than this critical value $T_{\min }$. To simplify we consider the case where $\gamma$ is constituted by characteristic lines. We consider $\left(\omega_{1}, \omega_{2}\right) \subset(0, L)$, and $L^{*}>L$ to avoid the critical time for observability. We assume that there exists two natural numbers $k, m$ such that

$$
L^{*}=k\left(\omega_{2}-\omega_{1}\right), \quad 2 \omega_{2}=m\left(\omega_{2}-\omega_{1}\right) .
$$

Let $n=\max \{m-1,2 k-m\}$. Consider the following periodic characteristic curve:

$$
\begin{aligned}
& \gamma(t)= \begin{cases}-t+\omega_{2}, & \text { if } t \in\left[0, \omega_{2}-\omega_{1}\right], \\
t+2 \omega_{1}-\omega_{2}, & \text { if } t \in\left[\omega_{2}-\omega_{1}, 2\left(\omega_{2}-\omega_{1}\right)\right],\end{cases} \\
& \gamma(t)=\gamma\left(t-j 2\left(\omega_{2}-\omega_{1}\right)\right) \quad \text { for all } t \in\left[(j-1) 2\left(\omega_{2}-\omega_{1}\right), j 2\left(\omega_{2}-\omega_{1}\right)\right], \quad \forall 2 \leq j \leq n .
\end{aligned}
$$


We define

$$
\begin{aligned}
I_{j} & =\left((j-1)\left(\omega_{2}-\omega_{1}\right), j\left(\omega_{2}-\omega_{1}\right)\right), \quad 1 \leq j \leq n, \\
U_{j} & =\left\{t-\gamma(t) \text { with } t \in I_{j}\right\}, \quad 1 \leq j \leq n, \text { and } j \text { odd. } \\
U_{j} & =\left\{t+\gamma(t) \text { with } t \in I_{j}\right\}, \quad 1 \leq j \leq n, \text { and } j \text { even. }
\end{aligned}
$$

Note that $V_{j}$ is reduced to an unique point for all $j \geq 1$, due to the fact that $\gamma(t)$ is a characteristic curve in $I_{j}$. It is very easy to check that $\gamma$ satisfies all the conditions in Theorem 2.4. The time of control is:

$$
T=\max \left\{2 \omega_{2}-\left(\omega_{2}-\omega_{1}\right), 2\left(L^{*}-\omega_{2}\right)\right\} .
$$

Note that, for $L^{*}=L$ we obtain the minimal time required for any ray to cross the curve $\gamma$ with an angle larger than $\pi$. Here we consider any $L^{*}$ larger than $L$.

\section{Observability}

The main results in this paper can be obtained from some inequalities for the uncontrolled wave equation. In this section we prove these inequalities.

Consider the system

$$
\begin{cases}\varphi_{t t}-\varphi_{x x}=0, & \text { in } 0<x<L, 0<t<T, \\ \varphi(0, t)=\varphi(L, t)=0, & \text { in } \quad 0<t<T, \\ \varphi(x, 0)=\varphi^{0}(x), \varphi_{t}(x, 0)=\varphi^{1}(x), & \text { in } 0<x<L .\end{cases}
$$

We assume that $\left(\varphi^{0}, \varphi^{1}\right) \in H_{0}^{1}(0, L) \times L^{2}(0, L)$. The following result holds:

Proposition 4.1. Assume that $\gamma$ satisfies the hypothesis of Theorem 2.1. Then, there exists a constant $c(\gamma)>0$ such that the solution $\varphi$ of (4.10) satisfies

$$
\int_{0}^{T}\left|\frac{\mathrm{d}}{\mathrm{d} t} \varphi(\gamma(t), t)\right|^{2} \mathrm{~d} t \leq c(\gamma)\left\|\left(\varphi^{0}, \varphi^{1}\right)\right\|_{H_{0}^{1} \times L^{2}}^{2}
$$

Moreover, if $\gamma$ satisfies the hypothesis of Theorem 2.4, then there exists a constant $C(\gamma)>0$ such that

$$
\left\|\left(\varphi^{0}, \varphi^{1}\right)\right\|_{H_{0}^{1} \times L^{2}}^{2} \leq C(\gamma) \int_{0}^{T}\left|\frac{\mathrm{d}}{\mathrm{d} t} \varphi(\gamma(t), t)\right|^{2} \mathrm{~d} t .
$$

Remark 4.2. Estimate (4.11) is a regularity result for the trace of the solution of the wave equation $\varphi(x, t)$ on the curve defined by the trajectory $\gamma$. This result cannot be obtained from classical arguments or semigroup theory.

Estimate (4.12) is an observability inequality which establishes that the total energy of the solutions of the wave equation can be estimated from the value of the solution $\varphi$ at $\gamma(t)$ for a large enough time interval $t \in(0, T)$.

The constant $C(\gamma)$ in (4.12) depends on the bounds for $\gamma^{\prime}, c_{1}$ and $c_{2}$. As we show in the proof, it blows up as $c_{1} \rightarrow 0$ or $c_{2} \rightarrow \infty$.

Proof. Note that it is enough to consider smooth solutions since for low regular ones we can argue by density. We first prove the estimate (4.11).

Note also that it suffices to prove this inequality in the particular case in which $\gamma \in C^{1}([0, T])$ and $1-\left|\gamma^{\prime}(t)\right|$ does not change the sign in $t \in[0, T]$. In the general case, for piecewise $C^{1}$ functions satisfying the hypothesis of the theorem, we can argue on each subinterval where the function is $C^{1}$ and then add the resulting inequalities. 
We observe that, in the one-dimensional wave equation, it is possible to change the variables $x$ by $t$ and $t$ by $x$ without altering the equation. Thus, D'Alembert formula can be used to obtain the solution $\varphi(x, t)$ in terms of the solution at one extreme, say $x=0$, instead of the data at $t=0$ as usual. Indeed, we have

$$
\varphi(x, t)=\frac{1}{2}[\varphi(0, t-x)+\varphi(0, t+x)]+\frac{1}{2} \int_{t-x}^{t+x} \varphi_{x}(0, s) \mathrm{d} s .
$$

If $\varphi$ is defined on $(x, t) \in[0, L] \times[0, T]$ this formula holds only for those values $(x, t)$ for which $0 \leq t-x \leq t+x \leq T$. However, we can extend the solution of the wave equation $\varphi$ to $(x, t) \in(0, L) \times(-\infty, \infty)$ and formula $(4.13)$ is still valid for the whole domain $(x, t) \in(0, L) \times(0, T)$. This is always possible because the wave equation with Cauchy data at $t=0$ is well-posed for $t \geq 0$ and $t \leq 0$.

In particular, taking into account the homogeneous Dirichlet boundary conditions in (4.10) we have

$$
\varphi(\gamma(t), t)=\frac{1}{2} \int_{t-\gamma(t)}^{t+\gamma(t)} \varphi_{x}(0, s) \mathrm{d} s .
$$

Therefore,

$$
2 \frac{\mathrm{d}}{\mathrm{d} t} \varphi(\gamma(t), t)=\left(1+\gamma^{\prime}(t)\right) \varphi_{x}(0, t+\gamma(t))-\left(1-\gamma^{\prime}(t)\right) \varphi_{x}(0, t-\gamma(t))
$$

and we have,

$$
\begin{aligned}
4 \int_{0}^{T}\left|\frac{\mathrm{d}}{\mathrm{d} t} \varphi(\gamma(t), t)\right|^{2} \mathrm{~d} t \leq & 2 \int_{0}^{T}\left(1+\gamma^{\prime}(t)\right)^{2}\left|\varphi_{x}(0, t+\gamma(t))\right|^{2} \mathrm{~d} t \\
& +2 \int_{0}^{T}\left(1-\gamma^{\prime}(t)\right)^{2}\left|\varphi_{x}(0, t-\gamma(t))\right|^{2} \mathrm{~d} t \\
\leq & 2 \sup _{t \in[0, T]}\left|1+\gamma^{\prime}(t)\right| \int_{0}^{T}\left|\varphi_{x}(0, t+\gamma(t))\right|^{2}\left|1+\gamma^{\prime}(t)\right| \mathrm{d} t \\
& +2 \sup _{t \in[0, T]}\left|1-\gamma^{\prime}(t)\right| \int_{0}^{T}\left|\varphi_{x}(0, t-\gamma(t))\right|^{2}\left|1-\gamma^{\prime}(t)\right| \mathrm{d} t .
\end{aligned}
$$

In order to simplify the two integrals on the right hand side we introduce a suitable change of variables. As we show below, this requires that $1-\left|\gamma^{\prime}(t)\right|$ does not change its sign in $t \in[0, T]$.

To fix ideas we assume that $1-\left|\gamma^{\prime}(t)\right| \geq 0$ in $t \in[0, T]$. The other case, i.e. when $1-\left|\gamma^{\prime}(t)\right| \leq 0$ in $t \in[0, T]$, requires a similar analysis and we omit it.

Let us introduce the set $\mathcal{I}_{1} \subset[0, T]$ defined by

$$
\mathcal{I}_{1}=\left\{t \in[0, T] \text {, such that } 1+\gamma^{\prime}(t) \neq 0\right\} .
$$

Note that the function $s(t)=t+\gamma(t)$ is injective in $\mathcal{I}_{1}$ and therefore it can be used as a change of variables for the first integral in the right hand side of (4.15). In this way, we have

$$
\begin{aligned}
\int_{0}^{T}\left|\varphi_{x}(0, t+\gamma(t))\right|^{2}\left|1+\gamma^{\prime}(t)\right| \mathrm{d} t & =\int_{\mathcal{I}_{1}}\left|\varphi_{x}(0, t+\gamma(t))\right|^{2}\left|1+\gamma^{\prime}(t)\right| \mathrm{d} t \\
& =\int_{\mathcal{I}_{1}+\gamma\left(\mathcal{I}_{1}\right)}\left|\varphi_{x}(0, s)\right|^{2} \mathrm{~d} s \leq \int_{\gamma(0)}^{T+\gamma(T)}\left|\varphi_{x}(0, s)\right|^{2} \mathrm{~d} s .
\end{aligned}
$$

Analogously, if we define $\mathcal{I}_{2} \subset[0, T]$ as

$$
\mathcal{I}_{2}=\left\{t \in[0, T], \text { such that } 1-\gamma^{\prime}(t) \neq 0\right\},
$$


then $s(t)=t-\gamma(t)$ is injective in $\mathcal{I}_{2}$ and therefore the second integral in the right hand side of (4.15) can be estimated as follows,

$$
\begin{aligned}
\int_{0}^{T}\left|\varphi_{x}(0, t-\gamma(t))\right|^{2}\left|1-\gamma^{\prime}(t)\right| \mathrm{d} t & =\int_{\mathcal{I}_{2}}\left|\varphi_{x}(0, t-\gamma(t))\right|^{2}\left|1-\gamma^{\prime}(t)\right| \mathrm{d} t \\
& =\int_{\mathcal{I}_{2}-\gamma\left(\mathcal{I}_{2}\right)}\left|\varphi_{x}(0, s)\right|^{2} \mathrm{~d} s \leq \int_{-\gamma(0)}^{T-\gamma(T)}\left|\varphi_{x}(0, s)\right|^{2} \mathrm{~d} s .
\end{aligned}
$$

Combining (4.15)-(4.17) we obtain,

$$
\begin{aligned}
4 \int_{0}^{T}\left|\frac{\mathrm{d}}{\mathrm{d} t} \varphi(\gamma(t), t)\right|^{2} \mathrm{~d} t & \leq 2 \max _{t \in[0, T]}\left|1+\gamma^{\prime}(t)\right| \int_{\gamma(0)}^{T+\gamma(T)}\left|\varphi_{x}(0, s)\right|^{2} \mathrm{~d} s+2 \max _{t \in[0, T]}\left|1-\gamma^{\prime}(t)\right| \int_{-\gamma(0)}^{T-\gamma(T)}\left|\varphi_{x}(0, s)\right|^{2} \mathrm{~d} s \\
& \leq 2\left(1+\max _{t \in[0, T]}\left|\gamma^{\prime}(t)\right|\right)\left(\int_{\gamma(0)}^{T+\gamma(T)}\left|\varphi_{x}(0, s)\right|^{2} \mathrm{~d} s+\int_{-\gamma(0)}^{T-\gamma(T)}\left|\varphi_{x}(0, s)\right|^{2} \mathrm{~d} s\right) \\
& \leq 4\left(1+\max _{t \in[0, T]}\left|\gamma^{\prime}(t)\right|\right) \int_{-\gamma(0)}^{T+\gamma(T)}\left|\varphi_{x}(0, s)\right|^{2} \mathrm{~d} s \leq C\left\|\left(\varphi^{0}, \varphi^{1}\right)\right\|_{H_{0}^{1} \times L^{2}}^{2},
\end{aligned}
$$

where

$$
C=4\left(1+\max _{t \in[0, T]}\left|\gamma^{\prime}(t)\right|\right)(T+\gamma(T)+\gamma(0)+2 L) .
$$

Here, the last inequality is the usual boundary direct inequality that can be obtained by classical multipliers techniques (see [12]). In fact, for any time interval $\left(t_{1}, t_{2}\right)$ the following holds:

$$
\int_{t_{1}}^{t_{2}}\left|\varphi_{x}(0, t)\right|^{2} d t \leq\left(t_{2}-t_{1}+2 L\right)\left\|\left(\varphi^{0}, \varphi^{1}\right)\right\|_{H_{0}^{1} \times L^{2}}^{2}
$$

To prove this inequality, we multiply the equation (4.10) by $(L-x) \varphi_{x}$ and integrate in $x \in(0, L)$. We easily obtain,

$$
0=\frac{1}{2} \int_{t_{1}}^{t_{2}} \int_{0}^{L}\left(\left|\varphi_{t}\right|^{2}+\left|\varphi_{x}\right|^{2}\right) d x d t-\left[\int_{0}^{L}(L-x) \varphi_{x} \varphi_{t} d x\right]_{t_{1}}^{t_{2}}-\int_{t_{1}}^{t_{2}} \frac{\left|\varphi_{x}(0, t)\right|^{2}}{2} d t .
$$

Therefore, taking into account the conservation of the energy, i.e. $\left\|\varphi(\cdot, t), \varphi_{t}(\cdot, t)\right\|_{H_{0}^{1} \times L^{2}}$ is constant in time, we have

$$
\begin{aligned}
\int_{t_{1}}^{t_{2}}\left|\varphi_{x}(0, t)\right|^{2} d t= & \int_{t_{1}}^{t_{2}} \int_{0}^{L}\left(\left|\varphi_{t}\right|^{2}+\left|\varphi_{x}\right|^{2}\right) d x d t+2 \int_{0}^{L}(L-x) \varphi_{x}(x, T) \varphi_{t}(x, T) d x \\
& -2 \int_{0}^{L}(L-x) \varphi_{x}(x, 0) \varphi_{t}(x, 0) d x \\
\leq & \left(t_{2}-t_{1}\right)\left\|\left(\varphi^{0}, \varphi^{1}\right)\right\|_{H_{0}^{1} \times L^{2}}^{2}+L \int_{0}^{L}\left(\left|\varphi_{x}(x, T)\right|^{2}+\left|\varphi_{t}(x, T)\right|^{2}\right) d x \\
& +L \int_{0}^{L}\left(\left|\varphi_{x}(x, 0)\right|^{2}+\left|\varphi_{t}(x, 0)\right|^{2}\right) d x \\
= & \left(t_{2}-t_{1}+2 L\right)\left\|\left(\varphi^{0}, \varphi^{1}\right)\right\|_{H_{0}^{1} \times L^{2}}^{2}
\end{aligned}
$$


Now, we prove the estimate (4.12). To clarify the proof we divide it in several steps:

Step 1. Our first objective is to prove the following inequality: there exist $C>0$ and $r<1$, independent of $j=0, \ldots, J$, such that

$$
\int_{U_{j}}\left|\varphi_{x}(0, t)\right|^{2} \mathrm{~d} t-r \int_{V_{j}}\left|\varphi_{x}(0, t)\right|^{2} \mathrm{~d} t \leq C \int_{I_{j}}\left|\frac{\mathrm{d}}{\mathrm{d} t} \varphi(\gamma(t), t)\right|^{2} \mathrm{~d} t .
$$

The analysis is slightly different depending on the sign of $\gamma^{\prime}$ and we consider separately the two different cases.

Case A. Assume that $t \in I_{j}$ with $j \leq j_{1}$. In this case $-c_{2} \leq \gamma^{\prime}(t) \leq-c_{1}<0$. From identity (4.14), we can estimate $\left|\varphi_{x}(0, t-\gamma(t))\right|$ as follows:

$$
\begin{aligned}
\left|\varphi_{x}(0, t-\gamma(t))\right|^{2}= & \left(\frac{1+\gamma^{\prime}(t)}{1-\gamma^{\prime}(t)} \varphi_{x}(0, t+\gamma(t))-\frac{2}{1-\gamma^{\prime}(t)} \frac{\mathrm{d}}{\mathrm{d} t} \varphi(\gamma(t), t)\right)^{2} \\
= & \left(\frac{1+\gamma^{\prime}(t)}{1-\gamma^{\prime}(t)}\right)^{2}\left|\varphi_{x}(0, t+\gamma(t))\right|^{2}+\left(\frac{2}{1-\gamma^{\prime}(t)}\right)^{2}\left|\frac{\mathrm{d}}{\mathrm{d} t} \varphi(\gamma(t), t)\right|^{2} \\
& -2 \frac{1+\gamma^{\prime}(t)}{1-\gamma^{\prime}(t)} \varphi_{x}(0, t+\gamma(t)) \frac{2}{1-\gamma^{\prime}(t)} \frac{\mathrm{d}}{\mathrm{d} t} \varphi(\gamma(t), t) \\
\leq & (1+a)\left(\frac{1+\gamma^{\prime}(t)}{1-\gamma^{\prime}(t)}\right)^{2}\left|\varphi_{x}(0, t+\gamma(t))\right|^{2} \\
& +\left(1+\frac{1}{a}\right)\left(\frac{2}{1-\gamma^{\prime}(t)}\right)^{2}\left|\frac{\mathrm{d}}{\mathrm{d} t} \varphi(\gamma(t), t)\right|^{2},
\end{aligned}
$$

for any $a>0$ to be chosen later. Here we have used Young's inequality.

Multiplying by $1-\gamma^{\prime}(t)$ and integrating in $t \in I_{j}$ we obtain,

$$
\begin{aligned}
\int_{I_{j}}\left|\varphi_{x}(0, t-\gamma(t))\right|^{2}\left(1-\gamma^{\prime}(t)\right) \mathrm{d} t \leq & (1+a) \int_{I_{j}}\left|\frac{1+\gamma^{\prime}(t)}{1-\gamma^{\prime}(t)}\right|\left|\varphi_{x}(0, t+\gamma(t))\right|^{2}\left|1+\gamma^{\prime}(t)\right| \mathrm{d} t \\
& +\left(1+\frac{1}{a}\right) \int_{I_{j}} \frac{4}{1-\gamma^{\prime}(t)}\left|\frac{\mathrm{d}}{\mathrm{d} t} \varphi(\gamma(t), t)\right|^{2} \mathrm{~d} t \\
\leq & (1+a) \sup _{t \in I_{j}}\left|\frac{1+\gamma^{\prime}(t)}{1-\gamma^{\prime}(t)}\right| \int_{I_{j}}\left|\varphi_{x}(0, t+\gamma(t))\right|^{2}\left|1+\gamma^{\prime}(t)\right| \mathrm{d} t \\
& +\left(1+\frac{1}{a}\right) \frac{4}{1+c_{1}} \int_{I_{j}}\left|\frac{\mathrm{d}}{\mathrm{d} t} \varphi(\gamma(t), t)\right|^{2} \mathrm{~d} t .
\end{aligned}
$$

In order to simplify the first integral on the right hand side we follow the argument used for the first integral on the right hand side of (4.15). Again, this requires that $1-\left|\gamma^{\prime}(t)\right|$ does not change its sign in $I_{j}$. In this way, we easily obtain,

$$
\int_{I_{j}}\left|\varphi_{x}(0, t+\gamma(t))\right|^{2}\left|1+\gamma^{\prime}(t)\right| \mathrm{d} t \leq \int_{V_{j}}\left|\varphi_{x}(0, s)\right|^{2} \mathrm{~d} s
$$

On the other hand, we simplify the integral on the left hand side of (4.19) with the change of variables $s=$ $t-\gamma^{\prime}(t)$,

$$
\int_{I_{j}}\left|\varphi_{x}(0, t-\gamma(t))\right|^{2}\left(1-\gamma^{\prime}(t)\right) \mathrm{d} t=\int_{U_{j}}\left|\varphi_{x}(0, s)\right|^{2} \mathrm{~d} s
$$


Therefore, combining (4.19)-(4.21) we obtain,

$$
\int_{U_{j}}\left|\varphi_{x}(0, t)\right|^{2} \mathrm{~d} t-(1+a) \sup _{t \in I_{j}}\left|\frac{1+\gamma^{\prime}(t)}{1-\gamma^{\prime}(t)}\right| \int_{V_{j}}\left|\varphi_{x}(0, t)\right|^{2} \mathrm{~d} t \leq\left(1+\frac{1}{a}\right) \frac{4}{1+c_{1}} \int_{0}^{T}\left|\frac{\mathrm{d}}{\mathrm{d} t} \varphi(\gamma(t), t)\right|^{2} \mathrm{~d} t .
$$

In order to have the inequality (4.18) we only have to check the uniform bound

$$
\sup _{t \in I_{j}}\left|\frac{1+\gamma^{\prime}(t)}{1-\gamma^{\prime}(t)}\right| \leq c<1
$$

for some constant $c>0$, for all $j=0, \ldots, j_{1}$. In fact, this estimate is easily checked from the hypothesis $-c_{2} \leq \gamma^{\prime}(t) \leq-c_{1}<0$.

Once we have estimate (4.23), we choose $0<a<1 / c-1$ in (4.22) and we obtain (4.18) with $r=(1+a) c$.

The constant $r$ satisfies $r=(1+a) c \rightarrow 1$ if $c_{1} \rightarrow 0$ or $c_{2} \rightarrow \infty$. As we show below, the observability constant in (4.12) depends on $1 /(1-r)$ and therefore it blows up as $c_{1} \rightarrow 0$ or $c_{2} \rightarrow \infty$.

Case B. Now, assume that $t \in I_{j}$ with $j>j_{1}$, i.e. $0<c_{1} \leq \gamma^{\prime}(t) \leq c_{2}<\infty$ for all $t \in I_{j}$. From identity (4.14), we estimate now $\left|\varphi_{x}(0, t+\gamma(t))\right|$ as follows,

$$
\begin{aligned}
\left|\varphi_{x}(0, t+\gamma(t))\right|^{2}= & \left(\frac{1-\gamma^{\prime}(t)}{1+\gamma^{\prime}(t)} \varphi_{x}(0, t-\gamma(t))+\frac{2}{1+\gamma^{\prime}(t)} \frac{\mathrm{d}}{\mathrm{d} t} \varphi(\gamma(t), t)\right)^{2} \\
= & \left(\frac{1-\gamma^{\prime}(t)}{1+\gamma^{\prime}(t)}\right)^{2}\left|\varphi_{x}(0, t-\gamma(t))\right|^{2}+\left(\frac{2}{1+\gamma^{\prime}(t)}\right)^{2}\left|\frac{\mathrm{d}}{\mathrm{d} t} \varphi(\gamma(t), t)\right|^{2} \\
& +2 \frac{1-\gamma^{\prime}(t)}{1+\gamma^{\prime}(t)} \varphi_{x}(0, t-\gamma(t)) \frac{2}{1+\gamma^{\prime}(t)} \frac{\mathrm{d}}{\mathrm{d} t} \varphi(\gamma(t), t) \\
\leq & (1+a)\left(\frac{1-\gamma^{\prime}(t)}{1+\gamma^{\prime}(t)}\right)^{2}\left|\varphi_{x}(0, t-\gamma(t))\right|^{2}+\left(1+\frac{1}{a}\right)\left(\frac{2}{1+\gamma^{\prime}(t)}\right)^{2}\left|\frac{\mathrm{d}}{\mathrm{d} t} \varphi(\gamma(t), t)\right|^{2}
\end{aligned}
$$

for any $a>0$ to be chosen later.

Multiplying by $1+\gamma^{\prime}(t)$ and integrating in $t \in I_{j}$ we obtain,

$$
\begin{aligned}
\int_{I_{j}}\left|\varphi_{x}(0, t+\gamma(t))\right|^{2}\left(1+\gamma^{\prime}(t)\right) \mathrm{d} t \leq & (1+a) \int_{I_{j}}\left|\frac{1-\gamma^{\prime}(t)}{1+\gamma^{\prime}(t)}\right|\left|\varphi_{x}(0, t-\gamma(t))\right|^{2}\left|1-\gamma^{\prime}(t)\right| \mathrm{d} t \\
& +\left(1+\frac{1}{a}\right) \int_{I_{j}} \frac{4}{1+\gamma^{\prime}(t)}\left|\frac{\mathrm{d}}{\mathrm{d} t} \varphi(\gamma(t), t)\right|^{2} \mathrm{~d} t .
\end{aligned}
$$

Therefore, arguing as in the previous case, we easily obtain,

$$
\int_{U_{j}}\left|\varphi_{x}(0, t)\right|^{2} \mathrm{~d} t-(1+a) \sup _{t \in I_{j}}\left|\frac{1-\gamma^{\prime}(t)}{1+\gamma^{\prime}(t)}\right| \int_{V_{j}}\left|\varphi_{x}(0, t)\right|^{2} \mathrm{~d} t \leq\left(1+\frac{1}{a}\right) \frac{4}{1+c_{1}} \int_{I_{j}}\left|\frac{\mathrm{d}}{\mathrm{d} t} \varphi(\gamma(t), t)\right|^{2} \mathrm{~d} t .
$$

Note that, in this case, we have the uniform bound $\sup _{t \in I_{j}}\left|\frac{1-\gamma^{\prime}(t)}{1+\gamma^{\prime}(t)}\right| \leq c<1$ for some constant $c>0$ and for all $j>j_{1}$. Then, as in the previous case, we can choose $a$ such that (4.18) holds.

Step 2. Here we obtain the following inequality

$$
(1-r) \int_{0}^{2 L}\left|\varphi_{x}(0, t)\right|^{2} \mathrm{~d} t \leq C \int_{0}^{T}\left|\frac{\mathrm{d}}{\mathrm{d} t} \varphi(\gamma(t), t)\right|^{2} \mathrm{~d} t .
$$


From (4.18) we obtain

$$
\begin{aligned}
\sum_{j=1}^{J} \int_{U_{j}}\left|\varphi_{x}(0, t)\right|^{2} \mathrm{~d} t-r \sum_{j=1}^{J} \int_{V_{j}}\left|\varphi_{x}(0, t)\right|^{2} \mathrm{~d} t & \leq C \sum_{j=1}^{J} \int_{I_{j}}\left|\frac{\mathrm{d}}{\mathrm{d} t} \varphi(\gamma(t), t)\right|^{2} \mathrm{~d} t \\
& \leq C \int_{0}^{T}\left|\frac{\mathrm{d}}{\mathrm{d} t} \varphi(\gamma(t), t)\right|^{2} \mathrm{~d} t
\end{aligned}
$$

On the other hand, the hypothesis (2.6) and (2.7) allow us to obtain,

$$
\sum_{j=1}^{J} \int_{U_{j}}\left|\varphi_{x}(0, t)\right|^{2} \mathrm{~d} t-r \sum_{j=1}^{J} \int_{V_{j}}\left|\varphi_{x}(0, t)\right|^{2} \mathrm{~d} t \geq \int_{W_{1}}\left|\varphi_{x}(0, t)\right|^{2} \mathrm{~d} t-r \int_{W_{2}}\left|\varphi_{x}(0, t)\right|^{2} \mathrm{~d} t .
$$

Then, taking into account the $2 L$-periodicity of the solutions of the wave equation (4.10) and the fact that the length of $W_{2}$ is lower than the length of $W_{1}$ we have

$$
\int_{W_{1}}\left|\varphi_{x}(0, t)\right|^{2} \mathrm{~d} t-r \int_{W_{2}}\left|\varphi_{x}(0, t)\right|^{2} \mathrm{~d} t \geq(1-r) \int_{W_{1}}\left|\varphi_{x}(0, t)\right|^{2} \mathrm{~d} t \geq(1-r) \int_{0}^{2 L}\left|\varphi_{x}(0, t)\right|^{2} \mathrm{~d} t .
$$

This inequality together with (4.26) allows us to obtain (4.25).

Step 3. Finally, inequality (4.12) is just a consequence of (4.25) and the following classical boundary inverse inequality for the wave equation (see [12]): for $T \geq 2 L$ there exists a constant $C>0$ such that

$$
\left\|\left(\varphi^{0}, \varphi^{1}\right)\right\|_{H_{0}^{1} \times L^{2}} \leq C \int_{0}^{2 L}\left|\varphi_{x}(0, t)\right|^{2} \mathrm{~d} t .
$$

This concludes the proof of Proposition 4.1.

\section{Existence OF WEAK SOLUTiOnS}

In this section we prove Theorem 2.1. This requires some estimates on the nonhomogeneous problem (2.3) that we state first.

Proposition 5.1. Assume that $\psi \in L^{1}\left(0, T ; H^{-1}(0, L)\right)$ and $\gamma$ a curve satisfying the hypothesis of Theorem 2.1. There exists a constant $c(\gamma)$ such that the solution $\varphi \in C\left([0, T] ; H_{0}^{1}(0, L)\right) \cap C^{1}\left([0, T] ; L^{2}(0, L)\right)$ of $(2.3)$ satisfies the folowing estimate,

$$
\int_{0}^{T}\left|\frac{\mathrm{d}}{\mathrm{d} t} \varphi(\gamma(t), t)\right|^{2} \mathrm{~d} t \leq c(\gamma)\|\psi(x, t)\|_{L^{1}\left(0, T ; L^{2}(0, L)\right)}^{2} .
$$

Assume now that $\psi(x, t)=\Psi_{t}(x, t)$, with $\Psi \in L^{2}\left(0, T ; L^{2}(0, L)\right)$, then the solution $\varphi$ of (2.3) satisfies $\varphi \in$ $C\left(0, T ; H_{0}^{1}(0, L)\right) \cap C^{1}\left(0, T ; L^{2}(0, L)\right)$. Moreover, there exists a constant $\tilde{c}(\gamma)>0$ such that

$$
\int_{0}^{T}\left|\frac{\mathrm{d}}{\mathrm{d} t} \varphi(\gamma(t), t)\right|^{2} \mathrm{~d} t \leq \tilde{c}(\gamma)\|\Psi(x, t)\|_{L^{2}\left(0, T ; H_{0}^{1}(0, L)\right)}^{2} .
$$

Let us complete the proof of Theorem 2.1 before proving this result. Let $\mathcal{L}(\psi)$ be the linear operator defined by the left hand side of $(2.5)$, i.e.

$$
\mathcal{L}(\psi)=\left\langle u^{1}(x), \varphi(x, 0)\right\rangle_{1}-\int_{0}^{L} u^{0}(x) \varphi_{t}(x, 0) \mathrm{d} x+\langle f(t), \varphi(\gamma(t), t)\rangle_{1}^{t} .
$$


From estimate $(5.28), \mathcal{L}(\psi)$ is continuous in $L^{1}\left(0, T ; L^{2}(0, L)\right)$ and it defines a unique $u \in L^{\infty}\left(0, T ; H^{-1}\right)$ satisfying

$$
\int_{0}^{T} \int_{0}^{L} u(x, t) \psi(x, t) \mathrm{d} x \mathrm{~d} t=\mathcal{L}(\psi), \quad \text { for all } \psi(x, t) \in L^{1}\left(0, T ; L^{2}(0, L)\right) .
$$

Therefore $u$ solves (2.5). Moreover, for some constant $C>0$,

$$
\|u\|_{L^{\infty}\left(0, T ; L^{2}(0, L)\right)} \leq C\left(\left\|u^{0}\right\|_{L^{2}(0, L)}+\left\|u^{1}\right\|_{H^{-1}(0, L)}+\|f\|_{\left(H^{1}(0, L)\right)^{\prime}}\right) .
$$

The continuity $t \rightarrow u(t, x)$ from $[0, T] \rightarrow L^{2}(0, L)$ is easily obtained by a density argument since the solutions of (1.1) with $L^{2}$-controls and smooth initial data satisfy $u \in C\left([0, T] ; L^{2}(0, L)\right)$ (see [13]).

Now we focus on the regularity for $u_{t}$. We consider in $(5.31) \psi(x, t)=\Psi_{t}(x, t)$ where $\Psi \in \mathcal{D}((0, T) \times(0, L))$, the space of $C^{\infty}$ functions with compact support. From Proposition 5.1 the map $\Psi \rightarrow \mathcal{L}\left(\Psi_{t}\right)$ is linear and continuous in $L^{2}\left(0, T ; L^{2}(0, L)\right)$. Thus, we deduce that $u_{t} \in L^{2}\left(0, T ; L^{2}(0, L)\right)$. This concludes the proof of Theorem 2.1.

Proof of Proposition 5.1. The main idea is to apply Duhamel's principle to reduce the nonhomogeneous problem (2.3) to a suitable homogeneous one with nonzero final data for which estimate (4.11) holds. We observe that $\varphi(x, t)$ can be written as

$$
\varphi(x, t)=\int_{t}^{T} U(x, t . s) \mathrm{d} s,
$$

where $U(x, t, s)$ is the solution of

$$
\begin{cases}U_{t t}-U_{x x}=0, & 0<x<L, \quad 0<t<s<T, \\ U(x, s, s)=0, & 0<x<L, \\ U_{t}(x, s, s)=\psi(x, s), & 0<x<L, \quad 0<s<T, \\ U(0, t, s)=U(L, t, s)=0, & 0<t<s<T .\end{cases}
$$

From Proposition 4.1 and the conservation of the energy, there exists a constant $c(\gamma)>0$ such that,

$$
\begin{aligned}
\int_{0}^{s}\left|\frac{\mathrm{d}}{\mathrm{d} t} U(\gamma(t), t, s)\right|^{2} \mathrm{~d} t & \leq c(\gamma)\left(\|U(\cdot, 0, s)\|_{H_{0}^{1}(0, L)}^{2}+\left\|U_{t}(\cdot, 0, s)\right\|_{L^{2}(0, L)}^{2}\right) \\
& =c(\gamma)\|\psi(\cdot, s)\|_{L^{2}(0, L)}^{2} .
\end{aligned}
$$

On the other hand, we observe that

$$
\frac{\mathrm{d}}{\mathrm{d} t} \varphi(\gamma(t), t)=\frac{\mathrm{d}}{\mathrm{d} t} \int_{t}^{T} U(\gamma(t), t, s) \mathrm{d} s=\int_{t}^{T} \frac{\mathrm{d}}{\mathrm{d} t} U(\gamma(t), t, s) \mathrm{d} s=\int_{0}^{T} \chi_{(t, T)}(s) \frac{\mathrm{d}}{\mathrm{d} t} U(\gamma(t), t, s) \mathrm{d} s,
$$

where $\chi_{(t, T)}(s)$ is the characteristic function of the interval $(t, T)$. Therefore, from Minkowski inequality we have

$$
\begin{aligned}
\left\|\frac{\mathrm{d}}{\mathrm{d} t} \varphi(\gamma(t), t)\right\|_{L^{2}(0, T)} & \leq \int_{0}^{T}\left\|\chi_{(t, T)}(s) \frac{\mathrm{d}}{\mathrm{d} t} U(\gamma(t), t, s)\right\|_{L^{2}(0, T)} \mathrm{d} s \\
& =\int_{0}^{T}\left\|\frac{\mathrm{d}}{\mathrm{d} t} U(\gamma(t), t, s)\right\|_{L^{2}(0, s)} \mathrm{d} s .
\end{aligned}
$$

Combining (5.32) and (5.33) we easily obtain the estimate (5.28).

Now we prove the second part of Proposition 5.1. Assume that $\psi(x, t)=\Psi_{t}(x, t)$, with $\Psi \in L^{2}\left(0, T ; L^{2}(0, L)\right)$. Following the previous argument based on Duhamel's principle we write

$$
\varphi(x, t)=\int_{t}^{T} V(x, t . s) \mathrm{d} s
$$


where $V(x, t, s)$ is the solution of

$$
\begin{cases}V_{t t}-V_{x x}=0, & 0<x<L, \quad 0<t<s<T \\ V(x, s, s)=-\Psi(x, s), & 0<x<L, \quad 0<s<T \\ V_{t}(x, s, s)=0, & 0<x<L, \quad 0<s, \\ V(0, t, s)=V(L, t, s)=0, & 0<t<s<T\end{cases}
$$

Note that $V \in C\left(0, T ; H_{0}^{1}(0, L)\right) \cap C^{1}\left(0, T ; L^{2}(0, L)\right)$ and therefore the same is true for $\varphi$.

From Proposition 4.1 and the conservation of energy we deduce

$$
\int_{0}^{s}\left|\frac{\mathrm{d}}{\mathrm{d} t} V(\gamma(t), t, s)\right|^{2} \mathrm{~d} t \leq c(\gamma)\|\psi(\cdot, s)\|_{H_{0}^{1}(0, L)}^{2} .
$$

On the other hand, we observe that

$$
\begin{aligned}
\frac{\mathrm{d}}{\mathrm{d} t} \varphi(\gamma(t), t) & =\frac{\mathrm{d}}{\mathrm{d} t} \int_{t}^{T} V(\gamma(t), t, s) \mathrm{d} s=\int_{t}^{T} \frac{\mathrm{d}}{\mathrm{d} t} V(\gamma(t), t, s) \mathrm{d} s+\Psi(\gamma(t), t) \\
& =\int_{0}^{T} \chi_{(t, T)}(s) \frac{\mathrm{d}}{\mathrm{d} t} V(\gamma(t), t, s) \mathrm{d} s+\Psi(\gamma(t), t) .
\end{aligned}
$$

Therefore,

$$
\left\|\frac{\mathrm{d}}{\mathrm{d} t} \varphi(\gamma(t), t)\right\|_{L^{2}(0, T)} \leq \int_{0}^{T}\left\|\frac{\mathrm{d}}{\mathrm{d} t} V(\gamma(t), t, s)\right\|_{L^{2}(0, s)} \mathrm{d} s+\|\Psi(\gamma(t), t)\|_{L^{2}(0, T)} .
$$

The first term on the right hand side can be bounded using (5.34). On the other hand, taking into account that $L^{\infty}(0, L) \subset H_{0}^{1}(0, L)$, we have

$$
\|\Psi(\gamma(t), t)\|_{L^{2}(0, T)} \leq\|\Psi\|_{L^{2}\left(0, T ; L^{\infty}(0, L)\right)} \leq\|\Psi\|_{L^{2}\left(0, T ; H_{0}^{1}(0, L)\right)} .
$$

From (5.34)-(5.36) we easily obtain the inequality (5.29).

\section{Controllability}

In this section we briefly describe how to obtain the controllability result stated in Theorem 2.4 from the observability result in Proposition 4.1. This is a straightforward application of HUM-method.

The first step is to characterize the controls of (1.1). Let us introduce the adjoint system

$$
\begin{cases}\varphi_{t t}-\varphi_{x x}=0 & \text { in } x \in(0, L), t \in(0, T) \\ \varphi(0, t)=\varphi(L, t)=0 & \text { in } t \in(0, T), \\ \varphi(x, T)=\varphi^{0}, \quad \varphi_{t}(x, T)=\varphi^{1}, & \text { in } x \in(0, L) .\end{cases}
$$

with $\left(\varphi^{0}, \varphi^{1}\right) \in H_{0}^{1}(0, L) \times L^{2}(0, L)$.

Multiplying the equations in (1.1) by $\varphi$ and integrating by parts we easily obtain, at least formally, the following identity:

$$
\left\langle u^{1}(x), \varphi(x, 0)\right\rangle_{1}-\int_{0}^{L} u^{0}(x) \varphi_{t}(x, 0) \mathrm{d} x+\langle f(t), \varphi(\gamma(t), t)\rangle_{1}^{t}=\left\langle u_{t}(x, T), \varphi^{0}(x)\right\rangle_{1}-\int_{0}^{L} u(x, T) \varphi^{1}(x) \mathrm{d} x,
$$

where $\langle\cdot, \cdot\rangle_{1}$ denotes the duality product in $H_{0}^{1}(0, L)$ and $\langle\cdot, \cdot\rangle_{1}^{t}$ the duality product in $H^{1}(0, T)$. Note that weak solutions must satisfy this identity. Therefore, a control $f$ can be characterized as a function $f(t)$ which satisfies the identity

for all $\left(\varphi^{0}, \varphi^{1}\right) \in H_{0}^{1}(0, L) \times L^{2}(0, L)$.

$$
\left\langle u^{1}(x), \varphi(x, 0)\right\rangle_{1}-\int_{0}^{L} u^{0}(x) \varphi_{t}(x, 0) \mathrm{d} x+\langle f(t), \varphi(\gamma(t), t)\rangle_{1}^{t} \mathrm{~d} t=0,
$$


Let us now introduce the functional $J: H_{0}^{1}(0, L) \times L^{2}(0, L) \rightarrow \mathbb{R}$ given by

$$
J\left(\psi^{0}, \psi^{1}\right)=\frac{1}{2} \int_{0}^{T}\left|\frac{\mathrm{d}}{\mathrm{d} t} \psi(\gamma(t), t)\right|^{2} \mathrm{~d} t+\left\langle u^{1}(x), \psi(x, 0)\right\rangle_{1}-\int_{0}^{L} u^{0}(x) \psi_{t}(x, 0) \mathrm{d} x,
$$

where $\psi$ is the solution of (6.37) associated to the final data $\left(\psi^{0}, \psi^{1}\right)$. For $T$ sufficiently large, this functional is continuous convex and coercive, due to the inverse inequality stated in (4.12). Therefore it has an unique minimizer $\left(\psi_{m}^{0}, \psi_{m}^{1}\right) \in H_{0}^{1}(0, L) \times L^{2}(0, L)$. The minimizer satisfies

$$
\int_{0}^{T} \frac{\mathrm{d}}{\mathrm{d} t} \psi_{m}(\gamma(t), t) \frac{\mathrm{d}}{\mathrm{d} t} \varphi(\gamma(t), t) \mathrm{d} t+\left\langle u^{1}(x), \varphi(x, 0)\right\rangle_{1}-\int_{0}^{L} u^{0}(x) \varphi_{t}(x, 0) \mathrm{d} x=0,
$$

for all $\left(\varphi^{0}, \varphi^{1}\right) \in H_{0}^{1}(0, L) \times L^{2}(0, L)$. Thus, a control is given by $f \in\left(H_{0}^{1}(0, T)\right)^{\prime}$ such that

$$
\langle f(t), \eta(t)\rangle_{1}^{t}=\int_{0}^{T} \frac{\mathrm{d}}{\mathrm{d} t} \psi_{m}(\gamma(t), t) \frac{\mathrm{d} \eta}{\mathrm{d} t}(t) \mathrm{d} t, \quad \text { for all } \eta \in H^{1}(0, T) .
$$

Acknowledgements. Supported by the Grant MTM2008-03541 of the MICINN.

\section{REFERENCES}

[1] S. Avdonin and S. Ivanov, Families of exponentials: The method of moments in controllability problems for distributed paramenter systems. Cambridge University Press (1995).

[2] A. Bamberger, J. Jaffre and J.P. Yvon, Punctual control of a vibrating string: Numerical analysis. Comput. Maths. Appl. 4 (1978) 113-138.

[3] C. Castro, Boundary controllability of the one-dimensional wave equation with rapidly oscillating density. Asymptotic Analysis 20 (1999) 317-350.

[4] C. Castro and E. Zuazua, Unique continuation and control for the heat equation from a lower dimensional manifold. SIAM J. Control. Optim. 42 (2005) 1400-1434.

[5] R. Dáger and E. Zuazua, Wave propagation observation and control in 1-d flexible multi-structures. Math. Appl. 50 (2006).

[6] S. Hansen and E. Zuazua, Exact controllability and stabilization of a vibrating string with an interior point mass. SIAM J. Control Optim. 33 (1995) 1357-1391.

[7] A. Khapalov, Controllability of the wave equation with moving point control. Appl. Math. Optim. 31 (1995) 155-175.

[8] A. Khapalov, Mobile point controls versus locally distributed ones for the controllability of the semilinear parabolic equation. SIAM J. Contol. Optim. 40 (2001) 231-252.

[9] A. Khapalov, Observability and stabilization of the vibrating string equipped with bouncing point sensors and actuators. Math. Meth. Appl. Sci. 44 (2001) 1055-1072.

[10] J.-L. Lions and E. Magenes, Non-homogeneous boundary value problems and applications I. Springer-Verlag (1972).

[11] J.-L. Lions, Some methods in the mathematical analysis of systems and their control. Gordon and Breach (1981).

[12] J.-L. Lions, Contrôlabilité exacte, stabilisation et perturbations de systèmes distribués. RMA 8 and 9, Tomes 1 and 2, Masson, Paris (1988).

[13] J.-L. Lions, Pointwise control for distributed systems, in Control and estimation in distributed parameter systems, edited by H.T. Banks. SIAM (1992). 